\title{
Laser Surgery
}

National Cancer Institute

\section{Source}

National Cancer Institute. Laser Surgery. NCI Thesaurus. Code C15268.

A type of operation using the cutting powers of laser beams from various sources. The use of a laser either to vaporize surface lesions or to make bloodless cuts in tissue. 